\title{
Lettering the Old-Fashioned Way
}

\author{
By Erica L. Wood-Bedi, Northern Illinois University
}

$\mathrm{n}$ the mid-twentieth century, much commercial lettering was done as freehand lettering without the use of stencils or patterns. However, sometimes mechanical lettering (standard uniform characters) was used for drawings, charts, maps, or signs. In these cases, a special pen was held in a scriber and guided by a template. According to Integrated Publishing (Integrated Publishing: Mechanical Lettering), mechanical lettering is used primarily when clear, legible, standardized lettering is required (e.g., for title blocks and notes on drawings, marginal data for special maps, briefing charts, titles on photographs, and signs).

The LEROY lettering set was a popular mechanical lettering template used by professionals from the 1940 s to the 1970s. It was invented by the Keuffel \& Esser Company. The laminated plastic had characters engraved on the front, with the lines serving as guide grooves for the writer. A number on the upper righthand side gave the height of the characters in thousandths of an inch. At the bottom-center was a zero to assist

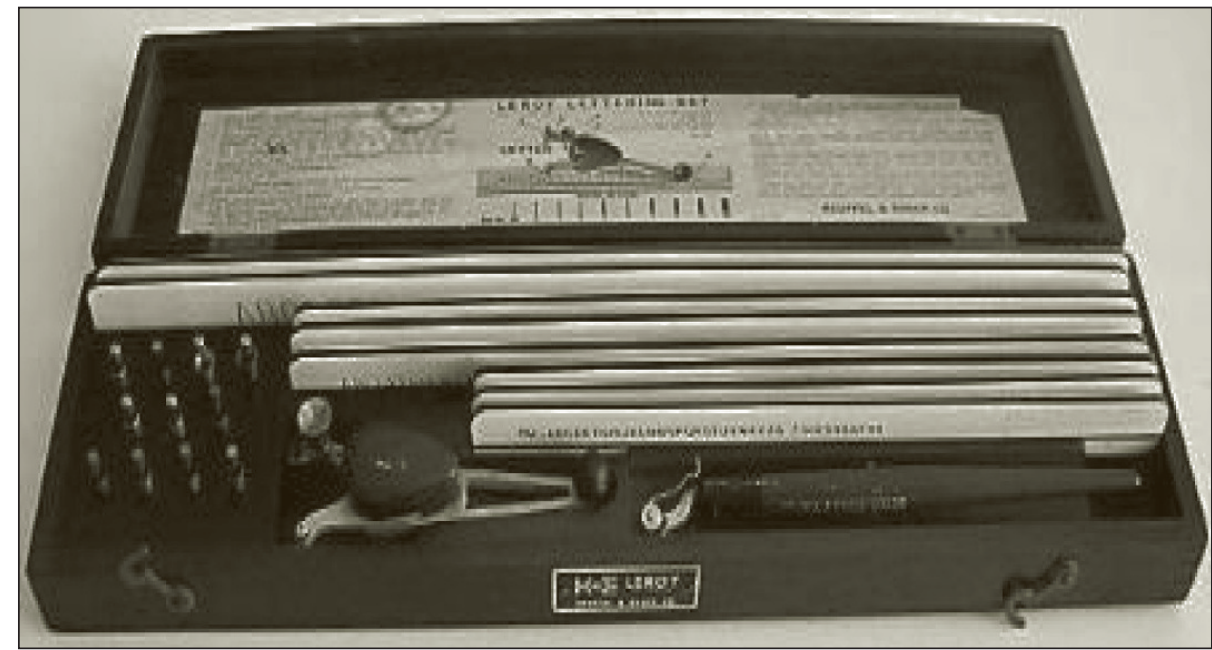

(Photo courtesy of http://patrickkerr.com/DRAFTINGEQUIP.html)

with proper spacing. LEROY scribers had two points: one for use with fine-groove templates and the other for wider-groove templates. The template was put along the top edge of a $\mathrm{T}$ square or the straight edge of a drafting machine (Integrated Publishing: Mechanical Lettering).

Vintage LEROY lettering sets can be found on the internet for \$119.95 (Vintage Drafting Equipment).

\section{References}

Integrated Publishing: Mechanical Lettering. Accessed 10 December, 2012 www.tpub.com/engbas/3-35.htm.

Vintage Drafting Equipment. Accessed 10 December, 2012 www.patrickkerr.com/ DRAFTINGEQUIP.html.

Vintage Drafting Equipment photo. Accessed 10 December, 2012 http://patrickkerr. com/DRAFTINGEQUIP.html 